\title{
Chemical analysis of secondary electron emission from a water cathode at the interface with a non-thermal plasma
}

Hernan E. Delgado ${ }^{1}$, Daniel T. Elg ${ }^{2}$, David M. Bartels ${ }^{3 *}$, Paul Rumbach ${ }^{4 *}$, David B. Go ${ }^{1,4 *}$

${ }^{1}$ Department of Chemical and Biomolecular Engineering, University of Notre Dame, Notre Dame, Indiana 46556 USA

${ }^{2}$ Department of Engineering, University of Southern Indiana, Evansville, Indiana, 47712 USA

${ }^{3}$ Radiation Laboratory and Department of Chemistry and Biochemistry, Notre Dame Radiation Laboratory, University of Notre Dame, Notre Dame, Indiana 46556 USA

${ }^{4}$ Department of Aerospace and Mechanical Engineering, University of Notre Dame, Notre Dame, Indiana 46556 USA

(Dated January 13, 2020)

\section{Supplementary Material}

\section{S.1 Molar conductivity $\left(\Lambda_{m}\right)$ of $\mathrm{NaClO}_{4}$}

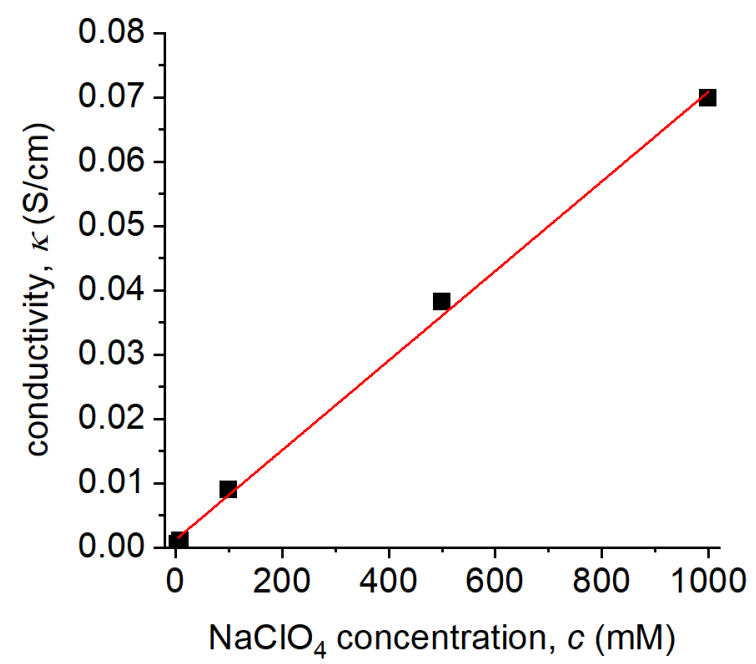

Figure S1. Measurement of conductivity $(\kappa)$ of an $\mathrm{NaClO}_{4}$ solution as a function of $\mathrm{NaClO}_{4}$ concentration $(c)$. The solid line is a curve fit producing an expression $\kappa=6.98 c$ with an adjusted coefficient of determination of $\mathrm{R}^{2}=0.99$.

\footnotetext{
*bartels.5@nd.edu,prumbach@nd.edu, dgo@nd.edu
} 


\section{S.2 Fit to obtain cell constants}

The cell constant was obtained as the slope from the linear regression of $V_{S} \Lambda_{m} / I$ with respect to the inverse of the $\mathrm{NaClO}_{4}$ concentration $(1 / c)$ under both positive and negative polarities. This approach derives from the expression of the system voltage,

$$
V_{S}=V_{P}+C_{c e l l}\left(\frac{I}{c \Lambda_{m}}\right),
$$

which, when slightly modified into

$$
\frac{V_{S} \Lambda_{m}}{I}=\frac{V_{P} \Lambda_{m}}{I}+C_{\text {cell }}\left(\frac{1}{c}\right),
$$

shows that the slope of the expression is the cell constant and the intercept leads to the plasma voltage $V_{p}$. The linear fit of the data is shown in Figure $\mathrm{S} 2$ for positive polarity (plasma anode) and in Figure S3 for negative polarity (plasma cathode). The cell constants are estimated as $25.5 \mathrm{~cm}^{-1}$ and $28.8 \mathrm{~cm}^{-1}$ for each configuration, respectively.

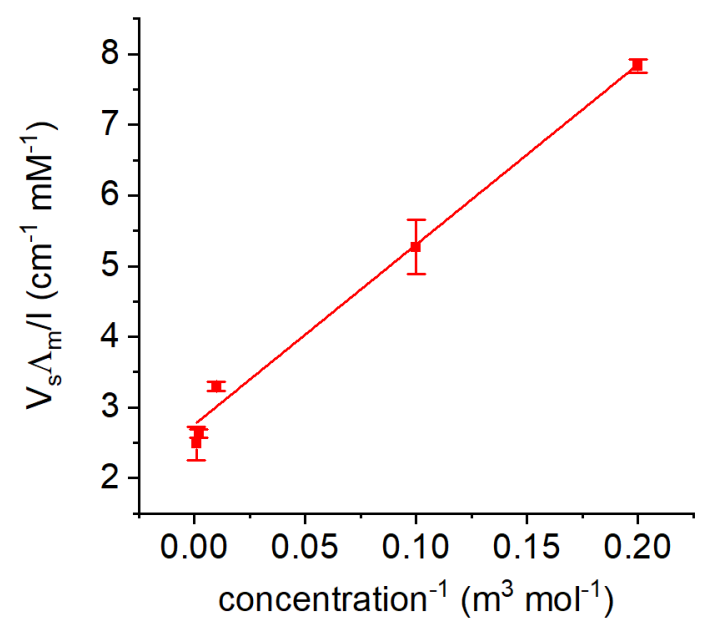

Figure S2. Plot of $V_{S} \Lambda_{m} / I$ as a function of the inverse of $\mathrm{NaClO}_{4}$ concentration in a plasma anode configuration. The solid line is a curve fit producing an expression $V_{S} \Lambda_{m} / I=25.5 c+2.8$ with an adjusted coefficient of determination of $\mathrm{R}^{2}=0.98$. Error bars reflect $95 \%$ confidence intervals for $N \geq 3$ experiments. 


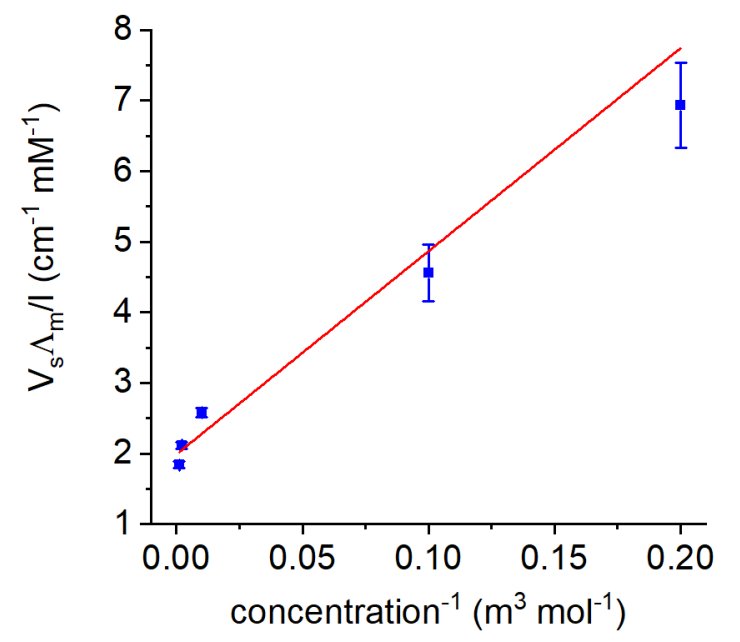

Figure S3. Plot of $V_{S} \Lambda_{m} / I$ as a function of the inverse of $\mathrm{NaClO}_{4}$ concentration in a plasma cathode configuration. The solid line is a curve fit producing an expression $V_{S} \Lambda_{m} / I=28.8 c+2.0$ with an adjusted coefficient of determination of $\mathrm{R}^{2}=0.70$. Error bars reflect $95 \%$ confidence intervals for $N \geq 3$ experiments.

\section{S.3 Extrapolation of the plasma voltage to estimate the cathode fall}

To estimate the voltage drop across the cathode fall for the plasma used in this configuration, the system voltage was measured at distances of $d=0.5,1,1.5$, and $2 \mathrm{~mm}$ between the metal capillary and a $100 \mathrm{mM} \mathrm{NaClO} 4$ solution. The data points were then linearly fitted, and the voltage drop across the cathode fall was estimated as the intercept (i.e., $d=0 \mathrm{~mm}$ ) similar to others' approach, ${ }^{1}$ yielding a value of 338 V. For all other experiments, the electrode distance was set at $d=1 \mathrm{~mm}$ and measured voltages were $\sim 360-370 \mathrm{~V}$, such that the cathode fall comprises at least $90 \%$ of the plasma voltage. 


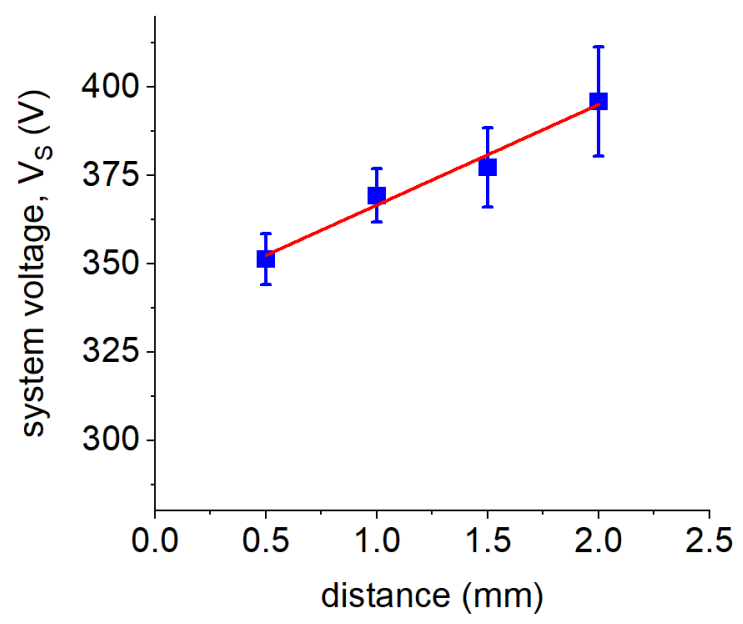

Figure S4. Plot of the system voltage, $V_{S}$, as a function of the distance between the metal capillary for a $100 \mathrm{mM} \mathrm{NaClO}_{4}$ solution in a plasma anode configuration. The solid line is a curve fit producing an expression $V_{S}=28.5 d+338$ with an adjusted coefficient of determination of $\mathrm{R}^{2}=$ 0.96. Error bars reflect $95 \%$ confidence intervals for $N \geq 3$ experiments.

\section{S.4 Detecting $e^{-}$aq in the plasma anode interface}

To confirm that $\mathrm{e}_{\text {aq }}^{-}$are present in the plasma anode configuration, we used the dissociative attachment reaction of $\mathrm{e}_{\text {aq }}^{-}$with chloroacetate and measured the ensuing chloride concentration with a chloride sensitive electrode as described in section 2.4 of the main manuscript. Our results are consistent with prior measurements by Goodman and Hickling ${ }^{2}$ with a minor difference. As in their experiments, our results show that $\mathrm{e}_{\text {aq }}^{-}$are present in a plasma anode configuration; however, we found that not all of the chloride production can be attributed to chloride abstraction by $\mathrm{e}_{\mathrm{aq}}^{-}$,

$$
e_{a q}^{-}+\mathrm{ClCH}_{2} \mathrm{CO}_{2}^{-} \rightarrow \cdot \mathrm{CH}_{2} \mathrm{CO}_{2}^{-}+\mathrm{Cl}^{-},
$$

as other species including $\bullet \mathrm{OH}$ are known to react with chloroacetate to produce chloride. ${ }^{3}$ In addition to experiments with pure chloroacetate, we repeated the same experiment using glycerol as an $\bullet \mathrm{OH}$ scavenger, which reacts with $\bullet \mathrm{OH}$ with a reaction rate constant of $1.8 \times 10^{10} \mathrm{M}^{-1} \mathrm{~s}^{-1},{ }^{4}$ and $\mathrm{NO}_{3}{ }^{-}$as an $\mathrm{e}^{-}$aq scavenger, which reacts with $\mathrm{e}_{\text {aq }}^{-}$with a reaction rate constant of $1.1 \times 10^{10}$ $\mathrm{M}^{-1} \mathrm{~S}^{-1} .5$ A summary of these results is shown in Figure S4. The chloride yield decreases roughly by about a third in the presence of $1 \mathrm{M} \mathrm{NO}_{3}{ }^{-}$as an $\mathrm{e}^{-}$aq scavenger, and it decreases by two-thirds when $1 \mathrm{M}$ glycerol is added as an $\bullet \mathrm{OH}$ scavenger. Overall, there seems to be about one solvated electron generated per incoming positive ion from the plasma. 
The hydroxyl radical, on the other hand, is more difficult to quantify, given that the exact mechanism and yield from chloroacetate to chloride is unknown. Another species that could potentially participate is $\mathrm{H} \bullet$, although some studies suggest that $\mathrm{H} \bullet$ does not produce $\mathrm{Cl}^{-}$from its reaction with chloroacetate, unless it is first converted to $\mathrm{e}^{-}$aq in an alkaline environment. ${ }^{6,7}$ Therefore, we believe that the portion of $\mathrm{Cl}^{-}$production not produced by $\mathrm{e}_{\text {aq }}^{-}$can be reasonably attributed to $\bullet \mathrm{OH}$, placing a minimum yield at $\sim 2 \cdot \mathrm{OH}$ per incoming argon ion. In one published study, the fraction of $\bullet \mathrm{OH}$ reacting to form $\mathrm{Cl}^{-}$(vs. $\mathrm{H}$ abstraction) was estimated at $20 \%,{ }^{3}$ which would correspond to a yield of $\sim 10 \bullet \mathrm{OH}$ per incoming ion. The exact yield for $\mathrm{e}^{-}$aq, $\mathrm{H} \bullet$, and $\bullet \mathrm{OH}$, however, is only marginally relevant to the problem at hand, and so, their detailed measurement must be studied more carefully.

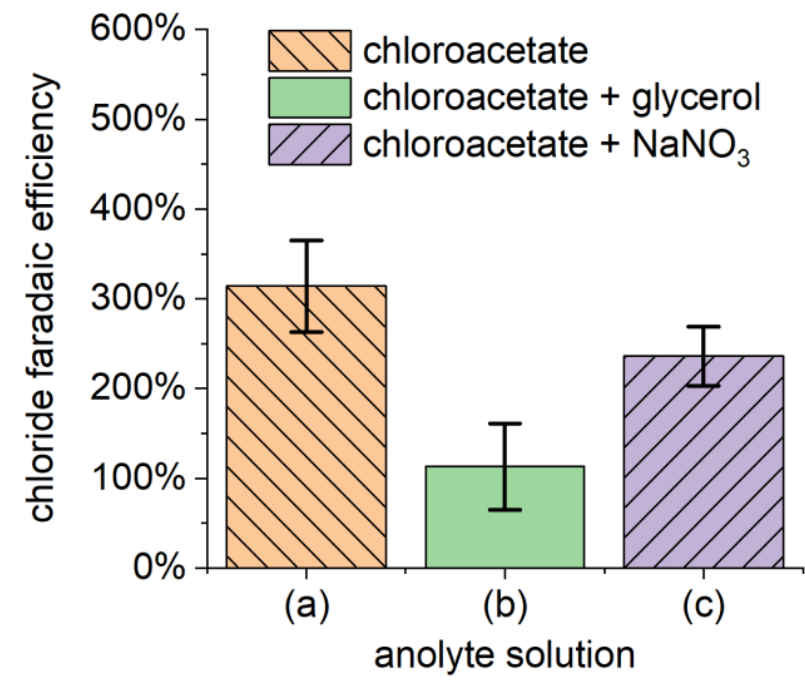

Figure S5. Measured chloride yield per unit charge (faradaic efficiency) after $10 \mathrm{~min}$ of plasma anode treatment for solutions consisting of (a) $1 \mathrm{M}$ chloroacetate, (b) $1 \mathrm{M}$ chloroacetate $+1 \mathrm{M}$ glycerol, and (c) $1 \mathrm{M}$ chloroacetate $+1 \mathrm{M}$ sodium nitrate. Error bars reflect 95\% confidence intervals for $N \geq 3$ experiments.

\section{S.5 Obtaining A and B coefficients}

The $A$ and $B$ gas coefficients used in the breakdown expression (eq 9 in the main text) were obtained from the intercept and slope, respectively, of the natural logarithm of Townsend's first ionization coefficient $(\alpha)$ plotted as a function of the inverse of the reduced electric field, $(E / p)^{-1}$. The first ionization coefficient was predicted from the solution of the Boltzmann equation using the online tool BOLSIG+ and cross-sections from the Phelps database. ${ }^{8,9}$ Results for a reduced electric field $(E / p)$ range of 3 to $70 \mathrm{~V}$ Torr ${ }^{-1} \mathrm{~cm}^{-1}$ are shown in Figure S5. The same methodology was used to obtain the $A$ and $B$ coefficients from different cross-section databases including 
SIGLO $^{10}$, TRINITI ${ }^{11}$, Morgan ${ }^{12}$, IST-LISBON ${ }^{13}$, and Biagi ${ }^{14}$, with each producing similar values as well as similar results for the secondary emission coefficient $\gamma$. The $A$ and $B$ coefficients for different argon/water gas compositions using the Morgan database ${ }^{12}$ were also obtained, yielding higher values for $A$ and $B$ in every case, which would correspond to lower $\gamma$ values.

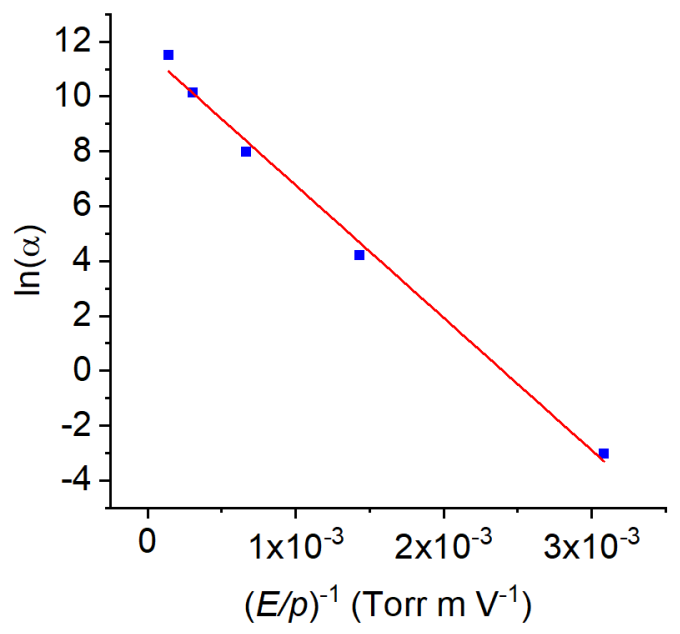

Figure S6. Natural logarithm of Townsend's first ionization coefficient $(\alpha)$ as a function of the inverse reduced electric field $(E / p)^{-1}$ over the $E / p$ range of 3 to $70 \mathrm{~V} \mathrm{Torr}{ }^{-1} \mathrm{~cm}^{-1}$. The solid line is a curve fit producing an expression $\ln (\alpha)=-4831(p / E)+11.6$ with an adjusted coefficient of determination of $\mathrm{R}^{2}=0.99$.

\section{References}

(1) Titov, V. A.; Rybkin, V. V.; Maximov, A. I.; Choi, H.-S. Characteristics of Atmospheric Pressure Air Glow Discharge with Aqueous Electrolyte Cathode. Plasma Chem Plasma Process 2005, 25 (5), 503-518. https://doi.org/10.1007/s11090-005-4996-z.

(2) Goodman, J.; Hickling, A.; Schofield, B. The Yield of Hydrated Electrons in GlowDischarge Electrolysis. Journal of Electroanalytical Chemistry and Interfacial Electrochemistry 1973, 48 (2), 319-322. https://doi.org/10.1016/S0022-0728(73)80272-4.

(3) Yokohata, A.; Ochmura, T.; Tsuda, S. Dechlorination Reaction in the Radiolysis of Aqueous Monochloroacetic Acid Solutions in the Presence of Nitrous Oxide. J. Phys. Chem. 1969, 73 (11), 4013-4014. https://doi.org/10.1021/j100845a075.

(4) Willson, R. L.; Greenstock, C. L.; Adams, G. E.; Wageman, R.; Dorfman, L. M. The Standardization of Hydroxyl Radical Rate Data from Radiation Chemistry. International Journal for Radiation Physics and Chemistry 1971, 3 (3), 211-220. https://doi.org/10.1016/0020-7055(71)90023-4.

(5) Anbar, M.; Alfassi, Z. B.; Bregman-Reisler, H. Hydrated Electron Reactions in View of Their Temperature Dependence. J. Am. Chem. Soc. 1967, 89 (5), 1263-1264. https://doi.org/10.1021/ja00981a041.

(6) Jortner, J.; Rabani, J. The Decomposition of Chloroacetic Acid in Aqueous Solutions by Atomic Hydrogen. II. Reaction Mechanisms in Alkaline Solutions. J. Phys. Chem. 1962, 66 (11), 2081-2084. https://doi.org/10.1021/j100817a003. 
(7) Matheson, M. S.; Rabani, J. Pulse Radiolysis of Aqueous Hydrogen Solutions. I. Rate Constants for Reaction of $\mathrm{e}_{\mathrm{aq}}{ }^{-}$with Itself and Other Transients. II. The Interconvertibility of eaq and $\mathrm{H}^{1}$. J. Phys. Chem. 1965, 69 (4), 1324-1335. https://doi.org/10.1021/j100888a037.

(8) Hagelaar, G. J. M.; Pitchford, L. C. Solving the Boltzmann Equation to Obtain Electron Transport Coefficients and Rate Coefficients for Fluid Models. Plasma Sources Sci. Technol. 2005, 14 (4), 722-733.

(9) Phelps Database, www.lxcat.net, Retrieved on November 5, 2019.

(10) SIGLO Database, www.lxcat.net, Retrieved on November 5, 2019.

(11) TRINITI Database, www.lxcat.net, Retrieved on November 5, 2019.

(12) Morgan Database, www.lxcat.net, Retrieved on November 5, 2019.

(13) IST-LISBON Database, www.lxcat.net, Retrieved on November 5, 2019.

(14) Biagi Database, www.lxcat.net, Retrieved on November 5, 2019. 